\title{
Methadon gegen Krebs - auch eine Frage für die Ethik und Theorie der Medizin
}

\author{
Eva Winkler
}

Online publiziert: 20. Oktober 2017

(C) Springer-Verlag GmbH Deutschland 2017

Kaum ein Thema hat über die letzten Wochen Krebspatienten und ihre behandelnden Ärzte in Deutschland bei Therapieberatungen mehr beschäftigt als der Einsatz von Methadon gegen Krebs. Die Berichterstattung über eine potentielle Anti-Tumorwirkung des Opioids in einer ARD-Sendung hat eine ungewöhnlich starke und langanhaltende Nachfrage nach der aus Drogenersatzprogrammen bekannten Substanz ausgelöst. In einer Online-Umfrage unter den Mitgliedern der hämatologischonkologischen Fachgesellschaft berichten über $80 \%$ der beinahe 500 teilnehmenden onkologisch tätigen Ärzte, dass sie von ihren Patienten oft oder sehr oft auf Methadon angesprochen werden. Etwa genauso viele berichten, dass die Gespräche auf Seiten der Patienten zu Enttäuschungen führen, weil Onkologen ganz überwiegend das Medikament, auf das die Patienten viel Hoffnung setzten, nicht rezeptieren (DGHO 2017).

Diese intensiv geführte Debatte, ihre Gründe und patientenseitige Wahrnehmung betreffen wesentliche Aspekte medizinischer Ethik, sodass wir das Thema im Rahmen unserer Fallrubrik in dieser Ausgabe diskutieren. Wie die meisten Situationen, die im klinischen Alltag moralisches Unbehagen auslösen, hat das Thema Methadon eine direkte einzelfallbezogene Relevanz und wird im Arzt-Patienten-Verhältnis verhandelt. Darüber hinaus verweist die Methadon-Debatte jedoch auf ethisch relevante Rahmenbedingungen und epistemologische Grundannahmen, die über die Reflexion des Einzelfalls hinaus ethisch bedenkens- und diskutierenswert sind.

Wie Stephan Sahm in seinem medizinethischen Kommentar darlegt, ist der Bewertungskonflikt beim Einsatz von Methadon als Krebsmedikament im Spannungsfeld zwischen dem informierten Therapiewunsch des Patienten und dem Nichtscha-

Prof. Dr. med. Dr. phil. E. Winkler ( $\triangle)$

Medizinische Onkologie, Nationales Centrum für Tumorerkrankungen (NCT), Im Neuenheimer

Feld 460, 69120 Heidelberg, Deutschland

E-Mail: eva.winkler@med.uni-heidelberg.de 
densprinzip angesiedelt, denn das Nebenwirkungsprofil von Methadon ist bekannt und relevant, während es für eine mögliche Wirkung gegen Krebszellen bislang nur Hinweise aus der Zellforschung gibt. Dieses Spannungsfeld mit dem Patienten auszuloten und gegebenenfalls aufzulösen, ist medizinethischer Alltag. Die Diskussion ist damit eine ähnliche, wie bei vielen anderen alternativen Therapieansätzen, die auf anekdotischen Erfolgsmeldungen oder uneindeutiger Datenlage basieren - nur dass bei vielen dieser Ansätze potentielle Schadensrisken nicht so gut untersucht und bekannt sind wie bei Methadon. Warum also ist im Fall Methadon die Nachfrage so vehement und warum die Enttäuschung so groß, wenn der Arzt am NichtSchadensprinzip festhält?

Die klinische Ethik muss die gesellschaftlichen Entwicklungen mit in den Blick nehmen, vor deren Hintergrund Entscheidungen am Krankenbett getroffen werden. Wesentlich scheinen in diesem Fall zwei Aspekte: Zum einen die wissenschaftstheoretische Frage, welche Art der Evidenz wir für ein Behandlungskonzept als ausreichend erachten, zum anderen das veränderte Informationsverhalten der Patienten in Zeiten der Digitalisierung.

Im Kern der Methadon-Diskussion geht es um die Bedingungen von begründetem Wissen. Sie rührt damit an die Grundlagen des Verständnisses von (ausreichender) Evidenz für die Nutzen- und Schadensbewertung, die für die medizinische Indikationsstellung Voraussetzung sind. Dass Ergebnisse aus der Petrischale nicht ausreichen, um einen Einsatz beim Menschen zu rechtfertigen, das ist auf den ersten Blick evident - letztlich sind die verschiedenen Phasen klinischer Prüfung im letzten Jahrhundert eingeführt worden, um Patienten vor schädlichen Nebenwirkungen zu bewahren und den Gebrauch unwirksamer Substanzen zu vermeiden.

Gleichwohl werden die herkömmlichen Methoden der evidenzbasierten Medizin derzeit durch die biomarkerstratifizierte Medizin auf den Prüfstand gestellt. Diese verspricht unter dem Label der „Personalisierten Medizin“ eine neue Ära, in der Patienten schneller zu einer für sie geeigneten Prävention, Diagnostik und Therapie kommen. Hinter der dabei angedeuteten Gegenüberstellung von personalisierten und den herkömmlichen evidenzbasierten Behandlungsansätzen, die man zunehmend auch in der medizinischen Fachliteratur findet, steht die Frage, ob die Mittel der evidenzbasierten Medizin ausreichen für den Nutzennachweis in stratifizierten und damit immer kleineren Subgruppen. Klar ist, dass es hierfür einer Erweiterung und Weiterentwicklung der Methodologie klinischer Studien bedarf, wie wir sie aktuell in der frühen klinischen Translationsforschung sehen. Wenn wir jedoch die Reaktion der Tumorzellen in einer Petrischale nicht als hinreichend überzeugend gelten lassen für den Einsatz von Methadon beim Menschen, sollten wir dann nicht auch kritischer auf den zurzeit verbreiteten Off-Label Einsatz zielgerichteter Substanzen allein aufgrund der molekularen Identifizierung von behandelbaren Mutationen in der Gewebeprobe des Patienten schauen? Oder gibt es gute Gründe, mechanistische Überlegungen auf molekularer Basis als hinreichende Evidenz zu bewerten gerade in sehr seltenen Konstellationen, in denen man nicht viele ähnliche Fälle für größere klinische Studien findet? Hier ist eine epistemologische Begleitung der Methodenentwicklung wünschenswert. Eine solche Begleitung hilft, die Qualität der Forschung auf dem Weg von der Zielstruktur auf Zellebene zum zielgerichteten Me- 
dikamenteneinsatz abzusichern, und sollte notwendige Wertsetzungen transparent machen.

Der zweite Aspekt ist das geänderte Verhalten der Patienten, die heute verschiedene Informationsquellen in Sachen Gesundheit nutzen - der Arzt ist, wenn überhaupt, nur eine davon. Entsprechend berichten fast alle Ärzte in der ambulanten Versorgung laut einer aktuellen Studie, dass sich das Informationsverhalten ihrer Patienten in den letzten fünf Jahren verändert hat und sich Patienten immer häufiger selbst zu medizinischen oder krankheitsbezogenen Fragen informieren (Bittner 2016) - ganz überwiegend im Internet und mit Hilfe sozialer Medien.

Zum Thema „Methadon gegen Krebs“ gibt es auf Facebook mehrere geschlossene Gruppen zwischen 800 und 5000 Mitgliedern, die Informationen austauschen zur Substanz selbst, aber auch zu Kliniken und Ärzten, die bereit sind, Methadon zu verschreiben, denn diese Praxis wird als „Wirkverstärker einer Chemo- oder Strahlentherapie" betrachtet.

In einer US-amerikanischen Studie zum Umgang von Patienten mit Online-Informationen standen zwei Drittel der Patienten ungeprüften Informationen aus dem Internet unkritisch gegenüber und überschätzten deren Glaubwürdigkeit und Richtigkeit (Murray 2003). Das Vertrauen in die Internet-Informationen war bei den Patienten eng mit der Nicht-Befolgung der ärztlichen Leitlinien und Behandlungsempfehlung korreliert und nicht überraschend auch mit angespannten Interaktionen im Arzt-Patienten-Gespräch. Es bedarf also auf Patientenseite einer besonderen kritischen Kompetenz, um die Qualität der online zugänglichen Gesundheitsinformationen beurteilen zu können: Diese umfasst nicht nur eine grundlegende Gesundheitskompetenz mit einem Verständnis dafür, wie die Medizin zu abgesichertem Wissen gelangt, sondern zusätzlich ein Vermögen, Online-Inhalte auf ihre Vertrauenswürdigkeit und Unabhängigkeit zu überprüfen. Doch schon diese grundlegende Gesundheitskompetenz ist in Deutschland bei mehr als der Hälfte der Bürger nur eingeschränkt vorhanden (Schaeffer et al. 2016). Damit haben über die Hälfte der Deutschen erhebliche Schwierigkeiten, gesundheitsrelevante Informationen zu finden, zu bewerten und damit gut informierte Entscheidungen zu treffen. Das bedeutet, dass neben der Verbesserung der Gesundheitskompetenz die Qualitätssicherung von Gesundheitsinformationen im Netz vor allem auf der Angebotsseite betrieben werden muss - durch wissenschaftlich abgesicherte Informationsangebote im Netz, beispielsweise dem Aufbau des nationalen digitalen Gesundheitsportals, wie es durch das Bundesministerium für Gesundheit geplant ist.

Da die Stärkung der Gesundheitskompetenz sowie die Qualitätssicherung im Netz erst in der ferneren Zukunft greifen werden, wird deutlich, welche Bedeutung der klassischen Vermittlungsleistung von Journalismus und Redaktionen der Massenmedien zukommt in der Aufklärung und im verantwortlichen Umgang mit „therapeutischen Durchbrüchen“. Daher haben wir einen zweiten Kommentar zur medienethischen Sicht auf die Methadon-Berichterstattung eingeladen, die ja in einem öffentlich-rechtlichen Sender ihren Ausgang nahm. Beatrice Dernbach benennt in ihrem Kommentar Defizite. Zum einen habe die TV-Berichterstattung Verschwörungstheorien Vorschub geleistet, statt über den notwendigen Prozess klinischer Prüfung aufzuklären. Vor allem aber fehlt es an Nachhaltigkeit in der Berichterstattung - während die Geschichte einer potentiellen Wirkung von Methadon gegen Krebs 
in mehreren TV-Formaten platziert wurde, wurden die kritischen Stellungnahmen der Fachgesellschaften nur in Printmedien noch einmal aufgenommen. Dernbach verweist aber auch auf die Verantwortung der vorgeschalteten Akteure aus dem Wissenschaftsbetrieb. Zu Recht kann man fragen, warum überhaupt die Wirkung von Methadon auf Zellkulturen schon eine Pressemitteilung wert ist und warum die Forscherin diese Geschichte für so relevant hält, dass sie durch mehrere TV-Sendungen tourt, um diesem Aspekt öffentliches Gewicht zu verleihen? Die Akademien der Wissenschaft haben in ihrem Statement zur Wissenschaftskommunikation 2014 und zum Umgang mit den Sozialen Medien 2016 die falschen Anreize zur Selbstvermarktung im Wissenschaftsbetrieb angeprangert und die Trennung von Fakten und Marketing gefordert. Es wird Zeit, dass sich hier Forschungsinstitutionen und Universitäten auf dem Wege der Selbstregulierung zu einer redlichen Kommunikation mit der Öffentlichkeit verpflichten, die insbesondere bei Forschung zu unheilbaren Erkrankungen die oft verzweifelte Situation der Adressaten berücksichtigt.

Fünf wissenschaftliche Fachgesellschaften haben in beispielhafter Weise und innerhalb kürzester Zeit zum Thema Methadon die Stimme der evidenzbasierten Medizin erhoben und mit fünf ähnlich lautenden Stellungnahmen öffentlich Position bezogen oder sich direkt an ihre Patienten gewendet. Das Thema birgt viele ethische Aspekte auf der klinischen wie gesellschaftlichen Ebene - mit der Fallrubrik in dieser Ausgabe möchte auch „Ethik in der Medizin“ einen gut begründeten Beitrag zur Debatte leisten.

\section{Literatur}

Bittner A (2016) Informierte Patienten und unzureichend vorbereitete Ärzte? Gesundheitsmonitor. Bertelsmann Stiftung, Gütersloh

Deutsche Gesellschaft für Hämatologie und Medizinische Onkologie (DGHO) (2017) Ergebnisse der DGHO Online-Umfrage „Methadon in der Krebstherapie“. https:/www.dgho.de/informationen/ stellungnahmen/gute-aerztliche-praxis/Methadon_Ergebnisse_Mitgliederumfrage_20170824.pdf.

Zugegriffen: 4. Okt. 2017

Murray E et al (2003) The impact of health information on the internet on health care and the physicianpatient relationship: national U.S. survey among 1.050 U.S. physicians. J Med Internet Res 5(3):e17

Schaeffer D, Vogt D, Berens EM, Hurrelmann K (2016) Gesundheitskompetenz der Bevölkerung in Deutschland - Ergebnisbericht. Universität Bielefeld, Bielefeld. https://doi.org/10.2390/0070-pub29088450 